\title{
DEVELOPMENT OF DYNAMic MODEl OF ROBOT WiTH PARAllel Structure BaSed ON 3D CAD Model
}

\author{
Vjekoslav Damic, Maida Cohodar \& Nedzma Kobilica
}
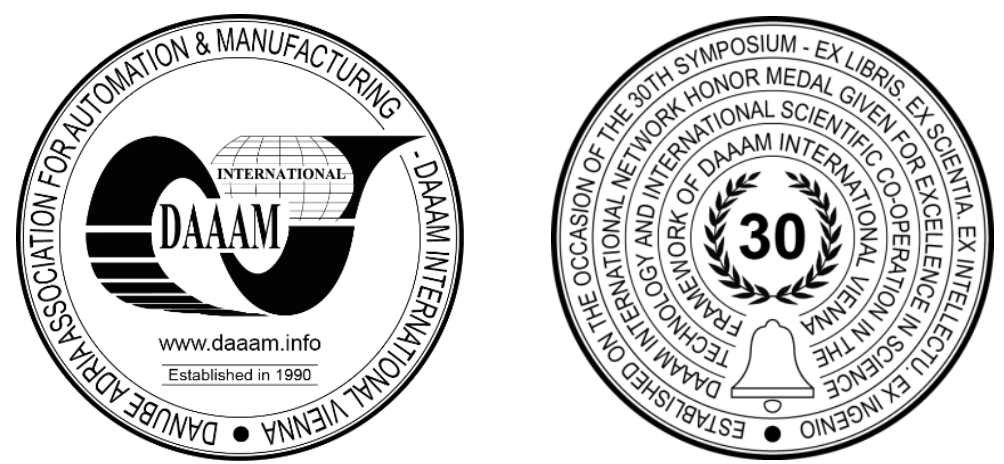

This Publication has to be referred as: Damic, V[jekoslav]; Cohodar, M[aida] \& Kobilica, N[edzma] (2019). Development of Dynamic Model of Robot with Parallel Structure Based on 3D CAD Model, Proceedings of the 30th DAAAM International Symposium, pp.0155-0160, B. Katalinic (Ed.), Published by DAAAM International, ISBN 9783-902734-22-8, ISSN 1726-9679, Vienna, Austria DOI: $10.2507 / 30$ th.daaam.proceedings.020

\begin{abstract}
Investigation of behaviour of parallel robot attracts the attention of researchers around the world. The main features of parallel robots are high precision and accuracy, work with high speed and large payload that make these robots capable of fast picking applications in agriculture, food and pharmaceutical industry, medicine etc. The most common limitation of the parallel robot is small workspace. Development of dynamic model of parallel robot based on its 3D CAD assembly is explained in the paper. Dynamic model of robot manipulator of ABB IRB 360 is automatically generated by importing of 3D CAD assembly from SolidWorks to Simulink programming environment. The obtained model can serve as a research platform to define task in joint or operational workspace, to investigate different control strategies, planning trajectories etc.
\end{abstract}

Keywords: Dynamic model; 3D CAD model; Parallel structure; Operational space.

\section{Introduction}

Popularity of robots with parallel structure increases during last time because of its good performances [1]. They can operate with high speed and accuracy, have good stiffness, large payload capacity to body-weight ratio and manipulability. Some types of Delta robots, its large potential, research directions and area of implementation are presented in [2]. Attention focused on the evaluation of the 3 DOF Delta parallel robot performances is given in [2]. Dynamic modelling of robot with parallel structure is subject of many researchers: dynamics of the Stewart platform is developed by bond graphs using Newton-Euler procedure in [3], a Delta parallel robot using the principle of the virtual work and the fundamental equations of the parallel robot dynamics in [6]. The new solution to the problem of torque minimization of the Delta robot has proposed in [7].

Attention of many researchers has paid to design and analysis of workspace and path planning of the parallel robots. The approach to compute a reachable workspace for the Delta robot is given in [6]. Path planning based on mapping the path to the joint space where the polynomial input method has adopted, is proposed in [9] providing the ability of the control all variables as well as moving to the target configuration along a smooth trajectory.

In [9], it has proved that all joints of the parallel manipulator system can move stably and accurately from initial to the target configuration within the specified time. 
Kinematic consideration of parallel robot is subject of [5], [6], [8] and [9]. Powerful 3D CAD software packages have opened up new perspectives for other disciplines and techniques (FEM, CAM, off-line programming of industrial robots etc.), making them more user friendly and more functional. Potential of 3D CAD model's use has recognized in processes of modelling and simulation of dynamic systems. Physical modelling of systems has implemented by bond graphs in [3], [4], [5] providing graphical representation of power flow on unique way regardless on physical domain from which subsystems come from. Virtual model of robot IRB360 is developed using software BondSimVisual in [5] while its kinematic model in other - BondSim. To start simulation, communication between these two models have to be provided and models exchange necessary information. Translator for importing mechanical CAD model to a simulation language source code of Modelica providing importing of the geometry, mass, inertia and constraints information from the drawing is given in [10]. The way how is Modelica integrated with Digital Mockup Tool using FMI is explained in [11]. To provide a trajectory tracking of the end effector of the Delta Robot, simplified dynamic equation is build and solved in MATLAB/Simulink [8]. Physical modelling of robot (uArm) with closed structure is implemented in [12] using MATLAB/Simulink/Simscape where model is obtained by connecting of the components, taken from the SimScape library, in a similar on way how they are is connected in the real robot. Simulation of the hexapod robot using MATLAB with the GUIDE graphical interface is presented in [13]. The forward and inverse kinematic problems of a parallel robot are implemented by MATLAB in [2].

Investigation of [10] are also supported by 3D CAD model, where one of two objectives is to introduce 3D robot (3D CAD) simulation for robotic workstations providing minimum chance of robot collision.

Unlike to [10], where by reading information from the mechanical assembly developed in any CAD software package the translator creates readable Modelica code, this paper described methodology of dynamic modelling of the parallel robot in MATLAB/Simulink environment also based on importing information from the mechanical assembly. But, in this case we obtained model composed from components reused from Simscape library. This paper is extension of [14]. Proposed procedure is explained on example of IRB 360. Structure of this robot is presented in Section 2. The procedure for translate 3D CAD assembly model of robot from SolidWorks to MATLAB/Simulink/Simscape programming environment is described in Section 3. Basic structure of developed dynamic model of manipulator is described and enhanced in Section 4. It is also explained one of ways to path planning. The paper finishes with conclusion and recommendations for the future work presented in Section 5.

\section{Structure of industrial robot IRB 360}

Structure of industrial robot ABB IRB360 is depicted in Fig.1. It is popular FlexPicker robot, as emphasised in [15], which in comparison to the hard automation provides more flexibility and shorter production cycles. It can operate with high speed, large payload and high accuracy. The robot consists of the fixed platform to which three upper arms are attached by three revolute joints. The lower arms are composed of two parallel bars; each of them is connected to the upper arm by two joints providing rotations about two axes. Finally, the moveable platform is attached to the lower arms by spherical joints and is always parallel with the fixed platform during its motion.

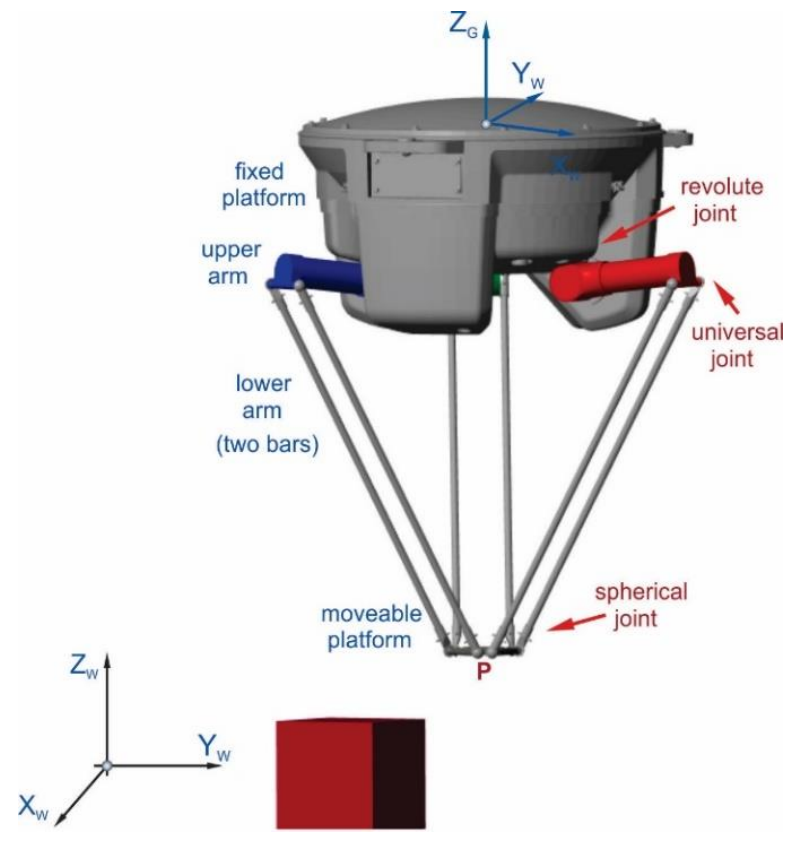

Fig. 1. Industrial robot ABB IRB 360

Coordinate frame $O_{G} X_{G} Y_{G} Z_{G}$ is fixed and attached to the upper platform and it is defined by manufacturer ABB. 


\section{3D CAD model of parallel robot}

To create mechanical assembly of IRB360, 3D CAD models of its parts (Fig. 2) are taken in form of SAT files from [15]. The robot assembly has developed using SolidWorks. In workspace of SolidWorks, the components the robot were loaded one by one. During this all parts are connected to each other providing the permitted motions between components as explained in Section 2. Parallelism between fixed and moveable platforms as well as bars of lower arms are also defined. That means the assembled model provides data about permitted motions between components as well as other constrains i.e. distances, angle constrains, parallelisms, etc. On other hand, the 3D CAD model of each part provides information about its geometry, mass centre, mass and inertia.

a)
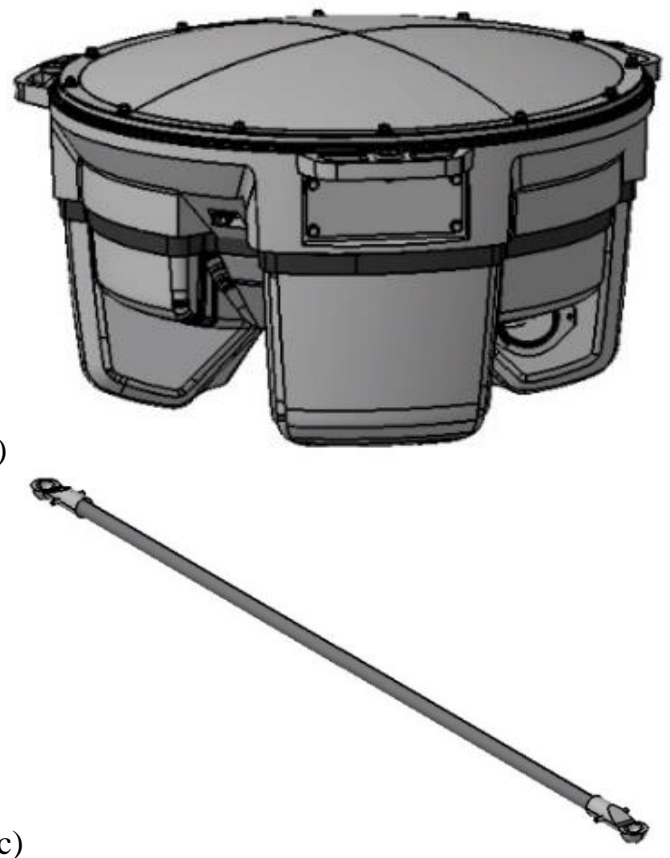

b)

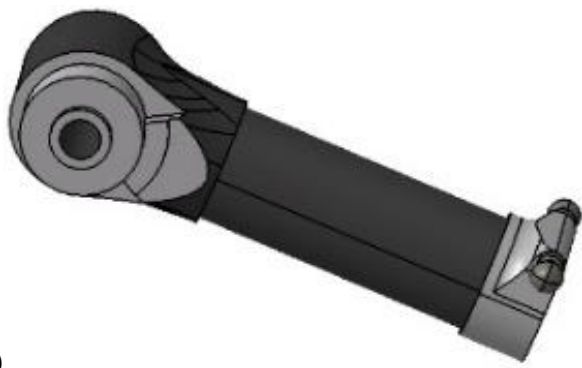

d)

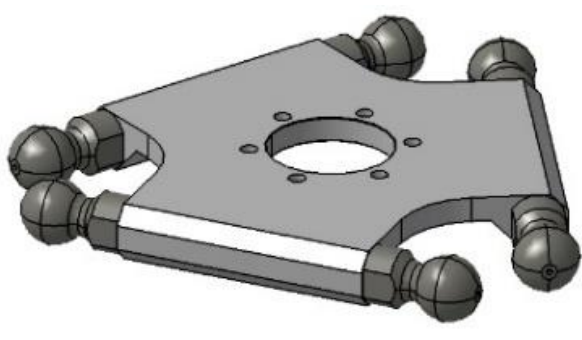

Fig. 2. ABB IRB 360 parts: a) Fixed platform, b) Upper arm, c) Bar of lower arm, d) Moveable platform

\section{Dynamic model of IRB360 in MATLAB/Simulink/Simscape environment}

MATLAB/Simulink provides automatically development of the multibody system dynamic model by importing 3D mechanical assembly model from the following software packages: SolidWorks, Autodesk Inventor and PTC Creo into Simulink environment. To translate mechanical assembly from 3D CAD software package into MATLAB/Simulink it has to be established a link - Simscape Multibody link plug-in - between these two software packages.

Using this link in SolidWorks, the mechanical assembly model has to be exported in the form of a XML file together with graphical files in this paper (irb360.slx). To import model in MATLAB, by typing instruction $>$ smimport('name of XML file') in MATLAB command line the dynamic model of mechanical assembly has been developed (in the form of SLX file). The generated dynamic model is composed of the Simscape library blocks devoted to physical modelling of the systems and it is often realized on one level; in our case it was realized in two levels in which models of the rigid bodies are developed separately as subsystems (on the second level).

In Simulink environment the model can be enhanced and we organized it systematically on more levels, Fig.3. Blocks from the left side provide following:

- Block World defines unique World coordinate frame,

- Block Mechanism Configuration sets gravity forces and

- Block Solver Configuration defines solver and provides its setting. We chose ode23tb based on TR-BDF2 method devoted for stiff models. It is implicit Runge-Kutta on two stages; the first is trapezoid rule step formula and second one is backward differentiation formula order 2 [17].

- Block Transform provides transformation from World to Global coordinate frames, Fig.1.

Model of manipulator is organized as a subsystem (large block in the middle of Fig.3 with mask defined by figure of IRB360). Its structure on the next level of decomposition is depicted in Fig.4. Manipulator is developed by blocks which represent joints: three revolute, six universal and six spherical and subsystems of rigid bodies, connected each to other according to structure of robot manipulator IRB360. To measure of rotation angle of three revolute joints in their properties it is specified sensing capability Position. Information about rotation angles are fed into Scope blocks. 


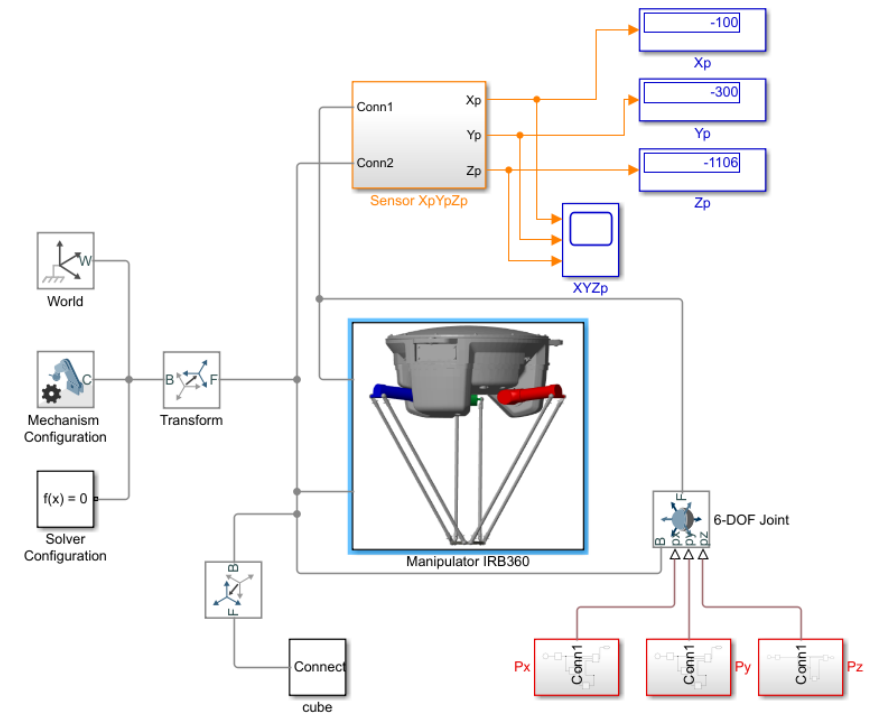

Fig. 3. Dynamic model of ABB IRB 360

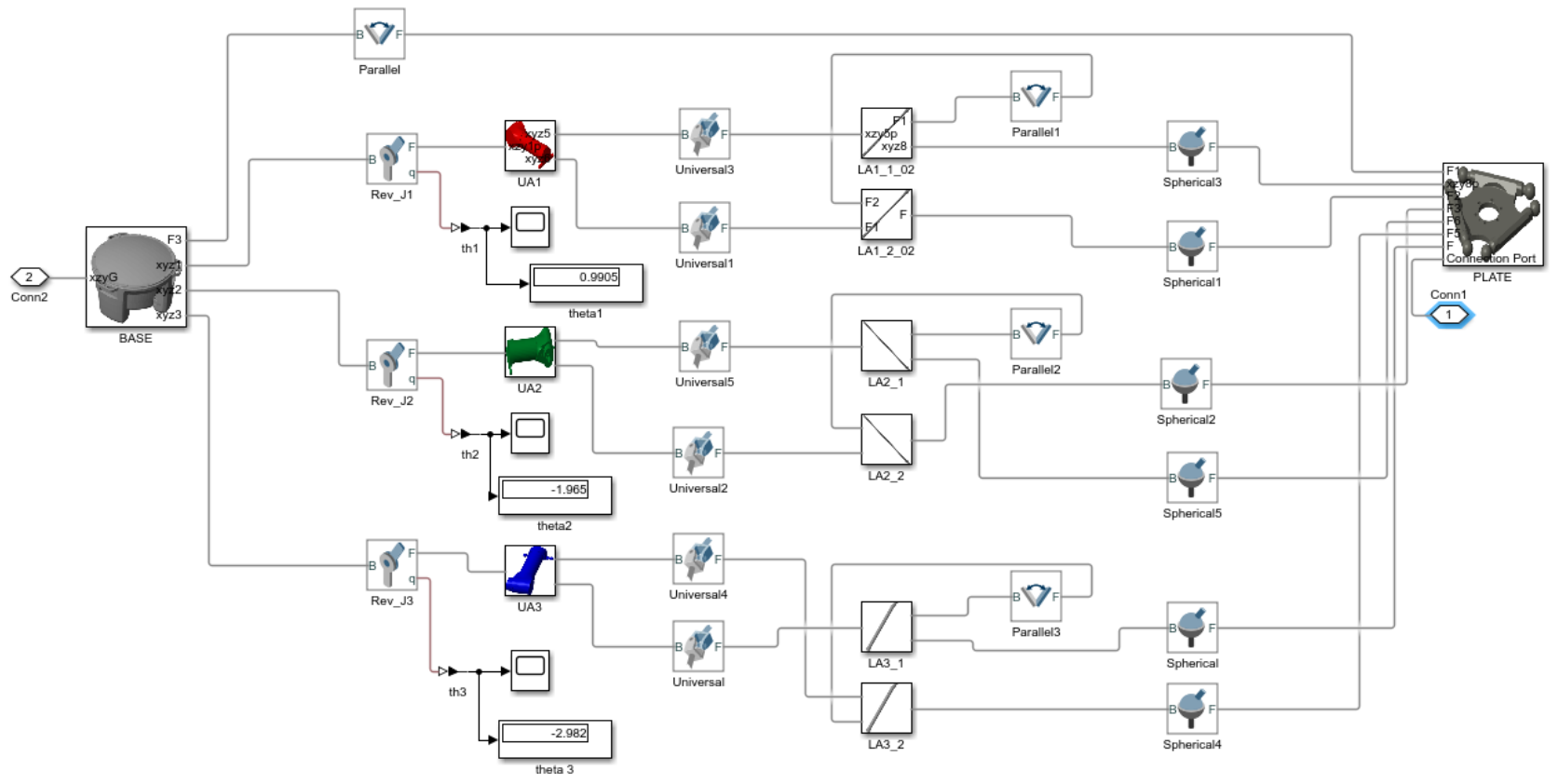

Fig. 4. Structure of subsystem manipulator IRB 360

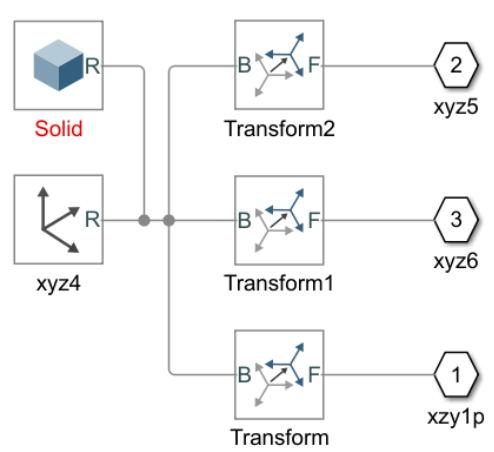

a)

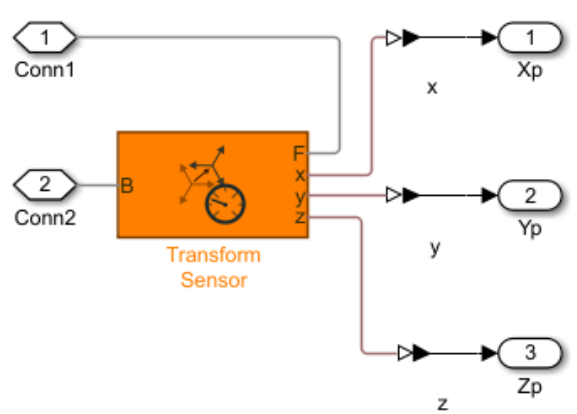

b)

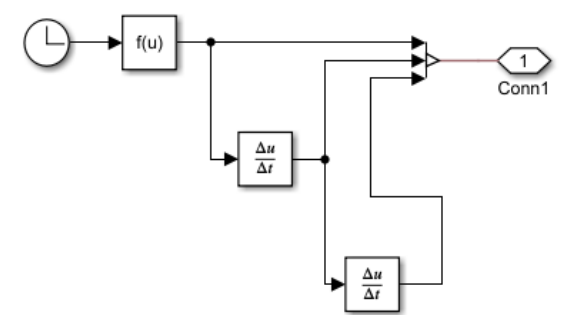

c)

Fig. 5. Structure of: a) subsystem of rigid body (UA1), b) block Sensor XpYpZp, c) $P x, P y$ and $P z$ 
The structure of rigid body subsystem is shown in Fig.5a. The rigid body is represented by block Solid, which defines the body shape from STL file generated during exporting model from SolidWorks. Data about mass, coordinate the mass centre, moment of inertia have also taken from 3D CAD model of body.

To measure coordinates of point $P$, located in the middle of moveable platform, block Transform Sensor is used which can measure the position of the origin and orientation of one coordinate frame with respect to the other. To this end; we attached the frame at the desired point $\mathrm{P}$ on the moveable platform and measure the coordinates of the origin in the global frame $O_{G} X_{G} Y_{G} Z_{G}$. Orientation of the platform does not change during the motion and we exclude possibility to measure the orientation changing.

To test the developed dynamic model a simple numerical experiment has been done. The frame attached to the moveable platform is connected to the global frame by a 6 DOF joint. That means the frame can moves by three translations and three rotations in the space. We enable three translations of point $P$ in the global frame. They are defined by blocks $P x, P y$ and $P z$ (Fig.5c). The platform moves from the initial posture to position when platform touches the cube (Fig.1). Law of motion of point $P$ is defined by quadratic functions to provide smooth change of velocity and accelerations of point $P$.

The trajectory of point $P$ is depicted in Fig.6a. This motion causes the change of joint angles in three revolute joints, Fig.6b.

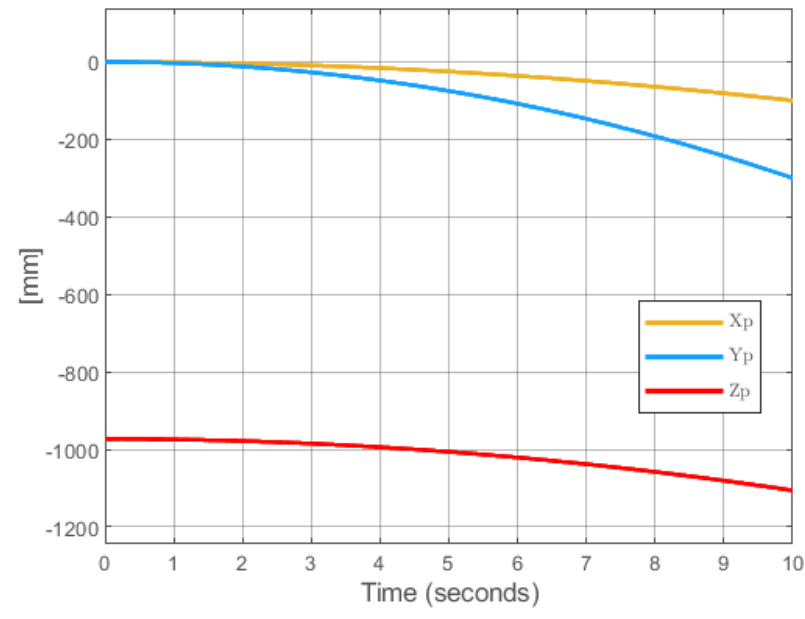

a)

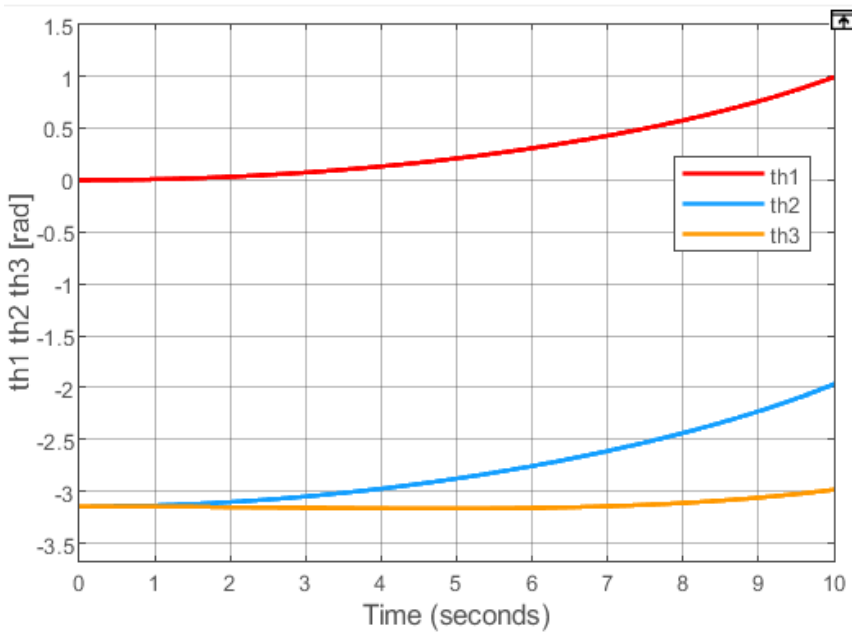

b)

Fig. 6. Time history of a) coordinate of point $P, \mathrm{~b}$ ) angle rotation th1, th2 and th3 in three revolute joints

\section{Conclusion}

Dynamic model of manipulator with parallel structure ABB IRB360 has been developed automatically by importing 3D CAD assembly model from SolidWorks to MATLAB/Simulink programming environment. This methodology simplifies the process of dynamic modelling and make it more comfortable. The model was enhanced by adding sensing and actuating blocks. The work task was defined by block 6DOF joint inserted between the frame attached to the point $P$ in the middle of the moveable platform and the global frame. In this block the law of motion of point $P$ with respect to the global frame was is defined.

The developed model can be used as a platform for investigation of different methods of the path planning, for improving and comparison of different control strategies, which we plan to do in the future work.

\section{References}

[1] Rey, L. \& Clavel, R. (1999). The Delta Parallel Robot, In: Parallel Kinematic Machine, C.R- Boer, (Ed.), (401-417), Springer-Verlang London Limited, DOI: 10.1007/978-1-4471-0885-6_29

[2] Stan, S.; Manic, M.; Szep, C. \& Balan, R. (2011). Performance analysis of 3 DOF Delta parallel robot, Proceedings of 4th International Conference on Conference Human System Interactions, DOI: 10.1109/HSI.2011.5937369

[3] Damic, V. \& Cohodar, M. (2015). Dynamic analysis of Stewart platform by bond graphs, Procedia Engineering, Vol.100, pp.226-233. (http://www.sciencedirect.com/science/article/pii/S1877705815003896)

[4] Damic, V. \& Cohodar, M. (2017). Multibody System Modeling, Simulation, and 3D Visualization, Chapter 17, pp.627-671, in book (C) Springer International Publishing Switzerland 2017, W. Borutzky (ed.), Bond Graphs for Modelling, Control and Fault Diagnosis of Engineering Systems, DOI 10.1007/978-3-319-47434-2_17

[5] Damic, V.; Cohodar, M. \& Voloder, A (2018). Modelling and Path Planning of Delta Parallel Robot in Virtual Environment, Proceedings of the 29th DAAAM International Symposium, pp.0149-0156, B. Katalinic (Ed.), 
Published by DAAAM International, ISBN 978-3-902734-20-4, ISSN 1726-9679, Vienna, Austria, DOI: 10.2507/29th.daaam.proceedings.021

[6] Staicu, S. (2008). Recursive modelling in dynamics of Delta parallel robot, Robotica (2009) volume 27, pp. 199207. (C) 2008 Cambridge University Press, doi:10.1017/S0263574708004451

[7] Baradat, C.; Arakelian, V.; Briot, S. (). Torque minimization of the Delta parallel robot, Available from: https://hal.archives-ouvertes.fr/hal-00451956 Accessed: 2019-10-15

[8] Prempraneerach, P. (2017). Workspace and Dynamic Trajectory Tracking of Delta Parallel Robot, Proceedings of International Computer Science and Engineering Conference (ICSEC), DOI 978-1-4799-4963-2/14/\$31.00 @2014 IEEE

[9] Li, L. (2018). Mechanism design and motion planning of parallel-chain nonholonomic manipulator, International Journal of Simulation Modelling, Vol.2, No.,17 (June 2018), 327-336, ISSN 1726-4529

[10] Engelson, V.; Bonus, P.; Popescu, L. \& Fritzson, P. (2003). Mechanical CAD with Multibody Dynamic Analysis Based on Modelica Simulation, Proceedings of of the 44th Scandinavian Conference on Simulation and Modeling (SIMS'2003), Västerås, Sweden, September 2003.

[11] Matsuda, S.; Toriya, H.; Suzuki, H. \& Ohtomi, K. (2017). Integration Modelica with Digital Mockup Tool using FMI, Proceedings of the 12th International Modelica Conference, May 15-17, 2017, Prague, Czech Republic, DOI $0.3384 / \mathrm{ecp} 17132547$

[12] Damic, V.; Cohodar, M. \& Tvrtkovic, M. (2016). Inverse Dynamic Analysis of Hobby Robot uArm by Matlab/Simulink, Proceedings of the 27th DAAAM International Symposium, pp.0095-0101, B. Katalinic (Ed.), Published by DAAAM International, ISBN 978-3-902734-08-2, ISSN 1726-9679, Vienna, Austria DOI: 10.2507/27th.daaam.proceedings.014

[13] Munoz, P.; Castano, B. \& R-Moreno, M.D. (2018). Simulation of the hexapod robot PINTO walking on irregular surface, International Journal of Simulation Modelling Vol.14, No. 17, 5-16, ISSN 1726-4529

[14] Kobilica, N. (2019). Dynamic modelling of industrial robot ABB IRB360, Master thesis, Faculty of Mechanical Engineering, University of Sarajevo, Sarajevo, Bosnia and Herzegovina

[15] https://new.abb.com/products/robotics/industrial-robots/irb-360. IRB 360 FlexPicker, Accessed on: 2019-10-15

[16] Supsomboon, S. \& Varodhomwathana, T. (2017). Robot and Plant Simulation for Automotive Part Production Process Design: A Case Study, International Journal of Simulation Modelling, Vol.16, No. 4, 617-629, ISSN 17264529.

[17] https://www.mathworks.com/help/simulink/ug/variable-step-solvers-in-simulink-1.html\#bsc6i5t Accessed on: 2019-10-15 